Article

\title{
High Performance Shape Memory Polyurethane Synthesized with High Molecular Weight Polyol as the Soft Segment
}

\author{
Manzoor Ahmad ${ }^{1}$, Bin Xu ${ }^{2}$, Hendra Purnawali ${ }^{3}$, Yongqing Fu ${ }^{2,4}$, Weimin Huang ${ }^{3}$, \\ Mohsen Miraftab ${ }^{1}$ and Jikui Luo ${ }^{1, *}$
}

1 Institute of Renewable Energy and Environment Technology, University of Bolton, Bolton BL3 5AB, UK; E-Mails: ma1cmr@bolton.ac.uk (M.A.); mm4@bolton.ac.uk (M.M.)

2 Department of Mechanical Engineering, School of Engineering and Physical Sciences, Heriot-Watt University, Edinburgh EH14 4AS, UK; E-Mails: binx@polysci.umass.edu (B.X.); richard.fu@uws.ac.uk (Y.F.)

3 School of Mechanical and Aerospace Engineering, Nanyang Technological University, 50 Nanyang Avenue 639798, Singapore; E-Mails: Purnawali@yahoo.com (H.P.); mwmhuang@ntu.edu.sg (W.H.)

4 Thin Film Centre, University of West of Scotland, Paisley PA1 2BE, UK

* Author to whom correspondence should be addressed; E-Mail: j12@bolton.ac.uk; Tel.: +44-120-4903-523; Fax: +44-120-4903-600.

Received: 18 April 2012; in revised form: 22 May 2012 / Accepted: 23 May 2012 /

Published: 31 May 2012

\begin{abstract}
Shape memory polyurethanes (SMPUs) are typically synthesized using polyols of low molecular weight $\left(\mathrm{M}_{\mathrm{W}} \sim 2,000 \mathrm{~g} / \mathrm{mol}\right)$ as it is believed that the high density of cross-links in these low molecular weight polyols are essential for high mechanical strength and good shape memory effect. In this study, polyethylene glycol (PEG-6000) with $\mathrm{M}_{\mathrm{W}} \sim 6000 \mathrm{~g} / \mathrm{mol}$ as the soft segment and diisocyanate as the hard segment were used to synthesize SMPUs, and the results were compared with the SMPUs with polycaprolactone PCL-2000. The study revealed that although the PEG-6000-based SMPUs have lower maximum elongations at break (425\%) and recovery stresses than those of PCL-based SMPUs, they have much better recovery ratios (up to 98\%) and shape fixity (up to 95\%), hence better shape memory effect. Furthermore, PEG-based SMPUs showed a much shorter actuation time of $<10 \mathrm{~s}$ for up to $90 \%$ shape recovery compared to typical actuation times of tens of seconds to a few minutes for common SMPUs, demonstrated their great potential for applications in microsystems and other engineering components.
\end{abstract}


Keywords: thermal-responsive SMPUs; polyurethanes; high molecular weight; low recovery stress

\section{Introduction}

Shape memory polymers (SMPs) have gained much attention owing to their special ability to return to their original shape upon external stimulation such as heating, light and chemicals [1,2]. The superior properties of lightweight, large shape recovery rate, low cost and easy processing as compared to shape memory alloys have promoted their rapid development and commercialization [3-5]. Shape memory polyurethane (SMPUs) are one emerging class of the SMPs [6-8]. They exhibit remarkable shape memory effect (SME), attributed to micro-phase segregation, which is interrelated to the chemical nature and processing methods used for synthesis. SMPUs typically consist of two phases: "fixed phase" and "reversible phase" which can also be termed as hard and soft segments, respectively [9,10]. The hard segments normally contain long sequences of hydrogen bonding sites and also serve as the physical cross-links [11]. These cross-link points prevent the neighbouring chains slipping from each other when it is subjected to deformation and consequential stress build-up [12]. These physical cross-links also act as net points during the shape recovery process. Therefore, the location of the cross-links is one of the most important factors affecting the SME and mechanical properties of the SMPs.

Literature study shows that physically cross-linked SMPUs possess better thermal and tensile properties [13]. Lee et al. found that SMPs with higher contents of cross-link points between the hard segments have higher shape recoverability than those with more contents of cross-link points at soft-hard segments [14]. The number of available physical cross-link points is related to the molecular weight, $\mathrm{M}_{\mathrm{w}}$, and the high hydroxyl number, $\mathrm{N}_{\mathrm{OH}}$, in the molecules (polyols and diisocyanates etc.), more specifically the polyols used in synthesizing the SMPUs. The polyols with lower molecular weights and higher hydroxyl number $\left(\mathrm{N}_{\mathrm{OH}}>50\right)$, normally have a larger number of cross-link points and stronger bonding owing to the short molecular chains, hence higher intermolecular interactions at soft segments, and vice versa for the polyols with high molecular weights due to the limited number of cross-link points [18].

Hydroxyl number of polyols is another important parameter which could affect the shape memory effect of the SMPUs significantly. Polyols with a higher functionality are more reactive, and can produce more cross-links, hence better mechanical properties, and vice versa. Up to now, most SMPUs were synthesized using polyols with low molecular weight ranging from a few hundreds to a few thousand $(200 \sim 3,000) \mathrm{g} / \mathrm{mol}$ and high functionality $\left(\mathrm{N}_{\mathrm{OH}}>50\right)$. Although some SMPUs have been synthesized using high molecular weight polycaprolactone (PCL) $\left(\mathrm{M}_{\mathrm{w}}\right.$ : 7,000 8,000 $\left.\mathrm{g} / \mathrm{mol}\right)$ polyol [15,16], polyethylene glycol (PEG-3400) and polytetramethylene glycol (PTMG-2900) as soft segments [17] and other SMPUs with varying molecular weight with good shape memory effect $[18,19]$, limited work has been done on SMPUs with high molecular weight polyols and low hydroxyl number, and little is known on how these affect the properties of the SMPUs. It is, therefore, scientifically interesting and important to investigate SMPUs with high $\mathrm{M}_{\mathrm{w}}$ and low $\mathrm{N}_{\mathrm{OH}}$ polyols. Furthermore, as the crystallinity, which affects the phase segregation significantly [13], of polyols with 
high and low molecular weight are different from each other, it is necessary to clarify how this affects the shape memory effects of the corresponding SMPUs due to their different crystalline natures.

Several SMPUs were recently synthesized with five types of polyols all having $\mathrm{M}_{\mathrm{w}} \sim 2,000$ and $\mathrm{N}_{\mathrm{OH}}=55-58$ [20]. At optimal concentrations and combinations of diisocyanates hard segments, these SMPUs have shown excellent properties with a maximum strain up to $1,000 \%$ and a shape recovery ratio of $95 \%$. In this study, a high molecular weight polyethylene glycol $\left(\mathrm{M}_{\mathrm{w}}=6,000\right.$ and $\left.\mathrm{N}_{\mathrm{OH}}=18\right)$ was chosen as the soft segment to synthesize the SMPUs, and their properties were compared with those polycaprolactone SMPUs (PCL-SMPU) with low molecular weight PCL polyol that have been published [20].

\section{Experiments}

\subsection{Synthesis of SMPUs}

In order to simplify the comparison, the chemicals and molar concentrations of the soft and the hard segments were the same as previously reported [20], except that the high molecular weight polyol, PEG-6000, was used as the soft segment in this work (hereafter denoted as PEG-SMPU series).

Through the process optimization, it was found that the optimal concentrations of the soft segment (polyols) and hard segment are 58 and $42 \mathrm{wt} \%$, respectively. The hard segment consists of 4,4'-diphenylmethane diisocyanate (MDI), isophorone diisocyanate (IPDI), PEG-200 and 1, 4-butanediol (BDO). The concentrations for the PEG-200 and BDO are 10 and $4.2 \mathrm{wt} \%$, respectively (Diisocyanate:PEG-200:BDO = 27.8:10.0:4.2\%). The molar ratio, $\gamma$, of MDI to IPDI [defined as $\gamma=\mathrm{MDI} /(\mathrm{MDI}+\mathrm{IPDI})]$ was varied from 1 to 0.325 as summarized in Table 1 , to synthesize different samples to clarify the effect of diisocyanates on the properties of SMPUs.

Table 1. Molar ratio of diisocyanates used for synthesis of shape memory polyurethane.

\begin{tabular}{|c|c|c|c|}
\hline Sample & MDI & IPDI & $\boldsymbol{\gamma}$ \\
\hline PEG-H1, PCL-S1 & 19.95 & 0 & 1.0 \\
\hline PEG-H2, PCL-S2 & 16.5 & 3.37 & 0.83 \\
\hline PEG-H3, PCL-S3 & 13.36 & 6.65 & 0.668 \\
\hline PEG-H4, PCL-S4 & 10.0 & 10.0 & 0.50 \\
\hline PEG-H5, PCL-S5 & 6.6 & 13.7 & 0.325 \\
\hline
\end{tabular}

$[\gamma=\mathrm{MDI} /(\mathrm{MDI}+\mathrm{IPDI})]$.

The synthesis process established is as follows: The set-up consisted of a $500 \mathrm{~mL}$ round bottom three-neck flask, a hot water bath with a temperature controller and an over-head stirrer. The flask was purged with a continuous flow of dry nitrogen and the temperature of the mixture was monitored by a thermometer. A measured amount of PEG-6000 and dimethylformamide (DMF) were first added into the flask, followed by IPDI and two drops of dibutyltin dilaurate catalyst. DMF is a solvent to dissolve the chemicals for reaction, and will vaporize during polymerization. After two hours of reaction at $90{ }^{\circ} \mathrm{C}$, the PEG-200 (Note: PEG-200 is part of the hard segment, not the polyol soft segment) was added into the flask, and was followed by the addition of MDI. The mixture was reacted for a further hour with continuous stirring. The process was completed by adding BDO, drop wise, as a chain 
extender for the chemical reaction at $60{ }^{\circ} \mathrm{C}$ for a further hour. The SMPU films were cast by pouring the polymer resin on a PTFE moulding plate. Then the films were baked at $60{ }^{\circ} \mathrm{C}$ for $12 \mathrm{~h}, 80{ }^{\circ} \mathrm{C}$ for $24 \mathrm{~h}$ and $100{ }^{\circ} \mathrm{C}$ for $8 \mathrm{~h}$, respectively, in a vacuum. The same method of synthesis for PCL-SMPUs was reported in the previous work [20].

\subsection{Characterization of Crystal Structure and Thermal Properties}

The synthesized SMPUs were characterized using a thermogravimetric analyzer (TGA) to assess thermal degradation, differential scanning calorimetry (DSC) to record thermal properties and wide-angle X-ray diffraction (WAXD) to determine crystalline structures of the polymers. Instron Universal Tester was used to test the tensile properties of the SMPUs.

For the TGA measurements, TA Instruments Q-500 was used with a heating rate of $10^{\circ} \mathrm{C} / \mathrm{min}$ and a temperature range of $25{ }^{\circ} \mathrm{C}$ to $800{ }^{\circ} \mathrm{C}$. Samples of about $10 \mathrm{mg}$ were put into the platinum sample holder, and the tests were conducted under a nitrogen gas flow. The thermal properties including transition temperature, $\mathrm{Tg}$, and heat of fusion were measured using Perkin Elmer DSC-7 with a temperature range of $20 \sim 150{ }^{\circ} \mathrm{C}$ at a heating rate of $10{ }^{\circ} \mathrm{C} / \mathrm{min}$. The typical weights of the specimens used for the analysis were between $10.5 \sim 15.0 \mathrm{mg}$.

D8 Discover X-ray diffractometer equipped with $\mathrm{Cu} \mathrm{K} \alpha$ radiation of a wavelength of $1.542 \AA$ was used to investigate the crystalline structures of the SMPUs, with a scanning angle $2 \theta$ between 5 to $40^{\circ}$ at $40 \mathrm{keV}$ and $40 \mathrm{~mA}$.

\subsection{Characterization of Shape Memory Properties}

Stress-strain, tensile strength, shape recovery and shape fixity were measured using Instron Universal Tester with a heat chamber. Dumb-bell shape specimens of $25 \mathrm{~mm}$ length and $7 \mathrm{~mm}$ width with an average thickness of $1.0 \mathrm{~mm}$ were prepared. For shape recovery and shape fixity measurements, the strain for all the samples was fixed at 50\%. The tests were performed with a crosshead speed of $50 \mathrm{~mm} / \mathrm{min}$ at temperatures above Tg. A typical thermomechanical cycle routine was used to characterize the behaviour of SMPUs [21,22]. The shape fixity, $R_{f}$, and shape recovery ratio, $R_{r}$, were calculated using the following equations,

$$
\begin{gathered}
\boldsymbol{R}_{\boldsymbol{f}}=\frac{\varepsilon_{\mathrm{u}}}{\varepsilon_{\mathrm{m}}} \times 100 \% \\
\boldsymbol{R}_{\boldsymbol{r}}=\frac{\varepsilon_{\mathrm{m}}-\varepsilon_{\mathrm{p}}(\mathrm{N})}{\varepsilon_{\mathrm{m}}-\varepsilon_{\mathrm{p}}(\mathrm{N}-1)} \times 100 \%
\end{gathered}
$$

Here $\varepsilon_{m}$ is the maximum strain applied, $\varepsilon_{u}$ the relaxed strain after cooling down with the force applied, and $\varepsilon_{p}$ is the residual strain after thermal recovery. $\mathrm{N}$ and $\mathrm{N}-1$ refer to the nth and nth -1 cycle for cyclic testing using the above defined stress, strain, temperature cycle.

\section{Results and Discussions}

\subsection{Thermal Property and Crystallinity}

Figure 1 shows the TGA curves for the PEG-SMPUs [23]. These SMPUs show three thermal transitions with corresponding weight loss clearly seen. The initial weight loss is around $60{ }^{\circ} \mathrm{C}$, 
corresponding to the evaporation of the water and solvents entrapped. The other two starting temperatures for material to decompose are around 250 and $300{ }^{\circ} \mathrm{C}$ for all the samples. The weight loss at $250{ }^{\circ} \mathrm{C}$ is rather small, and as the temperature increases to $\sim 300{ }^{\circ} \mathrm{C}$, it triggers another decomposition process with a large reduction in the weight. It is believed that these two decompositions correspond to soft and hard segments, respectively. As it shown in Figure 1, PEG-H1 has a slightly better stability than the others. The remaining weight above $410{ }^{\circ} \mathrm{C}$ is the residual materials such as carbon etc. The total weight losses up to $410{ }^{\circ} \mathrm{C}$ for sample PEG-H1, PEG-H2, PEG-H3, PEG-H4 and PEG-H5 are approximately 86, 88, 91, 90 and 94\% respectively, indicating the thermal stability decreases with increasing the IPDI contents.

Figure 1. Thermogravimetric analysis (TGA) curves of the PEG-SMPU samples.

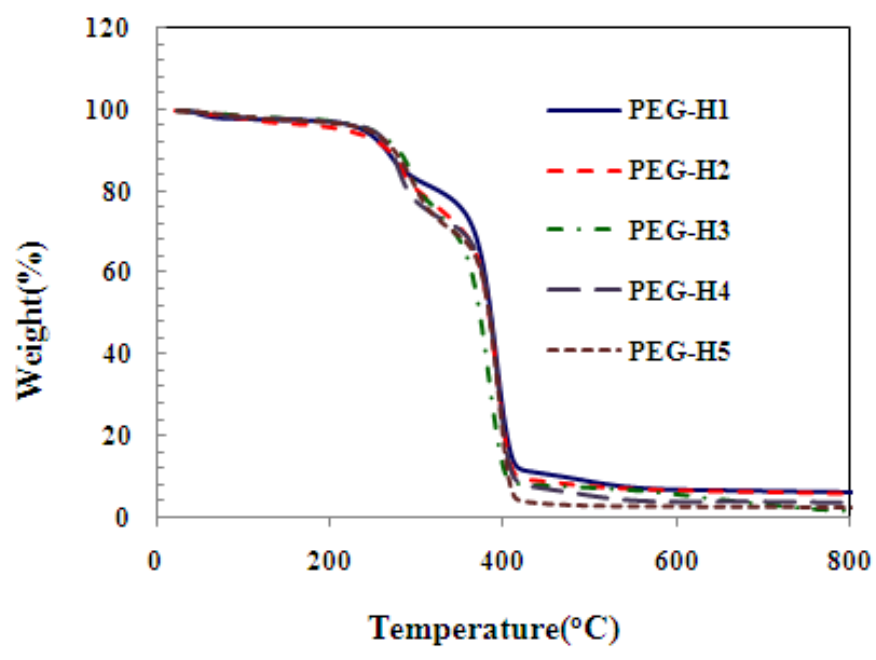

Table 2. Critical values of weight loss, $T_{m}$ and heat of fusion of PEG and PCL-SMPUs.

\begin{tabular}{cccccc}
\hline Sample & Weight Loss at $\left(\mathbf{4 1 0}{ }^{\circ} \mathbf{C}\right)$ & $\mathbf{T}_{\mathbf{m}}$ & $\Delta \mathrm{H}_{\mathrm{f}}$ & Stress at Break & Strain at Break \\
\hline PEG-6000 & $\mathbf{\%}$ & ${ }^{\circ} \mathbf{C}$ & $\mathbf{J g}^{-\mathbf{1}}$ & $\boldsymbol{M P a}$ & \% \\
PEG-H1 & N/A & $\mathbf{6 5 . 0}$ & $\mathbf{8 2 2 . 0}$ & N/A & N/A \\
PEG-H2 & 86 & 43.6 & 59.5 & 4.49 & 425 \\
PEG-H3 & 88 & 46.3 & 75.6 & 5.15 & 170 \\
PEG-H4 & 92 & 50.0 & 79.4 & 5.40 & 110 \\
PEG-H5 & 90 & 46.0 & 71.6 & 6.37 & 50 \\
PCL-2000 & 94 & 47.0 & 83.4 & 8.20 & 12 \\
PCL-S1 & N/A & $\mathbf{5 1 . 6}$ & $\mathbf{7 6 . 6}$ & N/A & N/A \\
PCL-S2 & 94 & 40.3 & 8.6 & 6.99 & 930 \\
PCL-S3 & 94 & 41.3 & 18.8 & 6.24 & 869 \\
PCL-S4 & 94 & 44.3 & 23.4 & 5.64 & 790 \\
PCL-S5 & 98 & 42.6 & 20.5 & 4.39 & 334 \\
\hline
\end{tabular}

The total weight loss for the PCL-SMPUs as listed in Table 2 is $94 \sim 98 \%$, higher than those of the PEG-SMPUs with the same molar ratios of MDI to IPDI. The PEG-SMPUs showed a slightly better thermal stability than the PCL-SMPUs, implying that the chemistry of polyol and the cross-link between the soft and the hard segments play important roles in thermal stability of the SMPUs. On the 
other hand, the weight loss was found to increase with the decrease of the molar ratio for both sample series, indicating that higher MDI concentration is better for thermal stability of the shape memory polymers.

Crystallization and melting of the soft segments in SMPUs are important in controlling the shape memory effect and deformation of the polymers, as the transition temperature of the SMPUs is normally dominated by the melting temperature, Tm [24]. As shown in Figure 2(a), a pure PEG-6000 polyol has a transition (melting) temperature from 58 to $68{ }^{\circ} \mathrm{C}$ with a narrow transition region of $\sim 10{ }^{\circ} \mathrm{C}$. Whilst a PCL polyol has a much broader transition region from 35 to $56{ }^{\circ} \mathrm{C}$ with a broad shoulder at about $40{ }^{\circ} \mathrm{C}$ (Figure 3(a)). The heat of fusion $\left(\Delta \mathrm{H}_{\mathrm{f}}\right)$ of the PEG-6000 polyol is about 822 $\mathrm{J} / \mathrm{g}$, more than one order of magnitude higher than $77 \mathrm{~J} / \mathrm{g}$ for the PCL-2000 polyol, implying that the PEG-6000 polyol has a high degree of crystallinity.

Figure 2. Differential scanning calorimetry (DSC) results of the PEG-6000 polyol (a) and PEG-SMPUs (b).
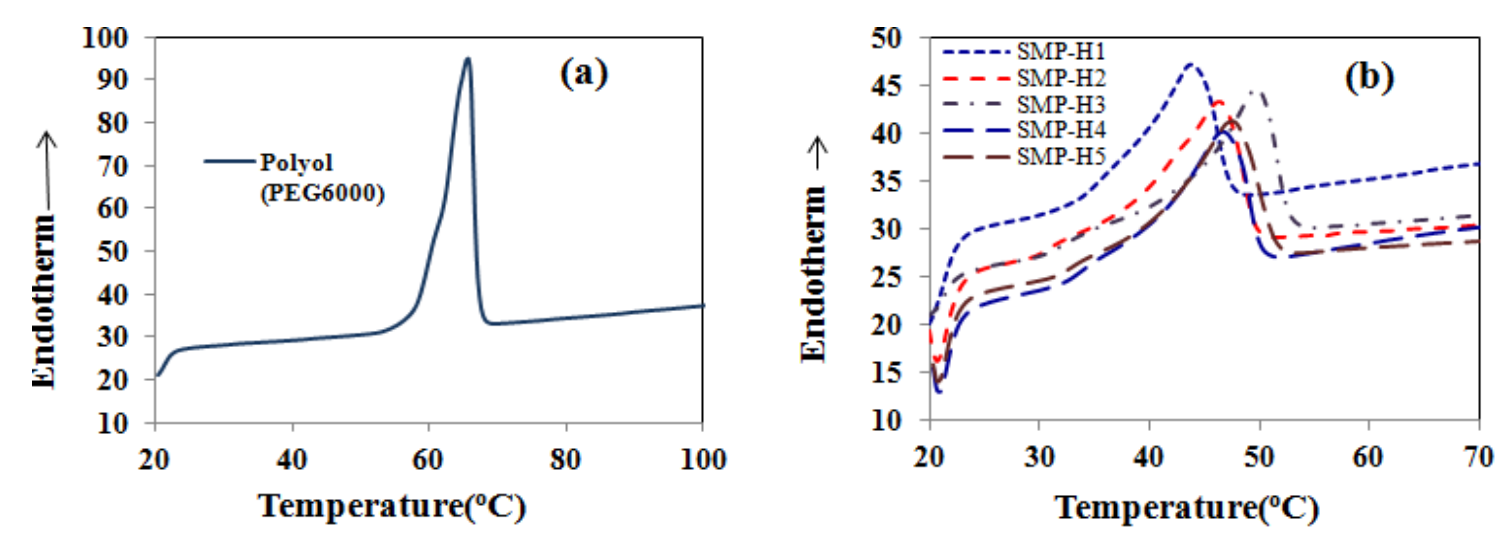

Figure 3. DSC curves $\Delta \mathrm{H}_{\mathrm{f}}\left(\mathbf{J g}^{-\mathbf{1}}\right)$ of the PCL-2000 polyol (a) and PCL-SMPUs (b).
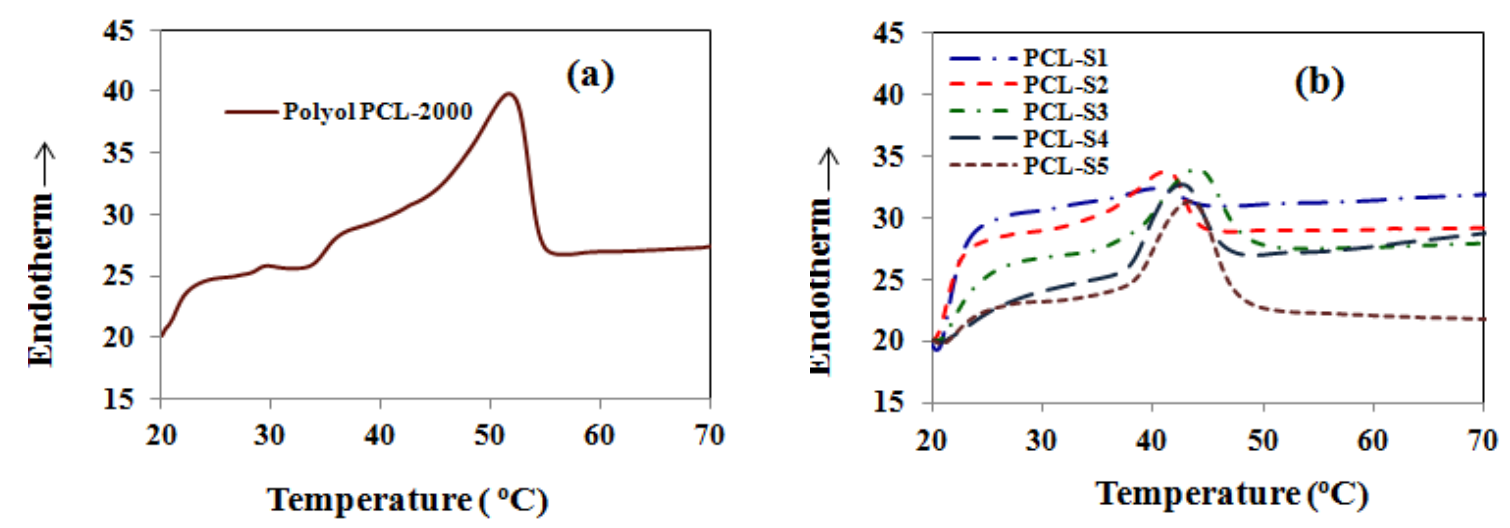

Figures 2(b) and 3(b) show the DSC curves of the SMPUs with PEG and PCL polyols at different molar ratios of $\gamma$. Once the polyols were mixed with the hard segments, the transition temperature decreased for both sample series, and the decrease of Tg was larger for the samples with higher MDI concentrations; with further decreasing the molar ratio to $\gamma=0.5$ or 0.325 , the transition temperature slightly increased as summarized in Table 2. As can be seen, similar to the low molecular weight polyol SMPUs developed, the high molecular weight polyol PEG-SMPUs have transition temperatures lower than $50{ }^{\circ} \mathrm{C}$, which are close to human body temperature and hence are suitable for implants and other medical applications [25,26]. 
Table 2 also summarizes the DSC measurement results for the PEG and PCL-based SMPUs and corresponding pure polyols. Comparison between the pure polyol PEG-6000 and PEG-SMPUs revealed a dramatic decrease in $\Delta \mathrm{H}_{f}$ from 822 to less than 100 once the hard segment was introduced. It is known that low heat of fusion is an indication of low crystallinity and high phase compatibility between the hard and the soft segments [9]. Decrease of $\Delta \mathrm{H}_{f}$ in the PEG-SMPUs is more drastic than in the PCL-SMPUs; comparison of the $\Delta \mathrm{H}_{f}$ of the PEG-SMPUs with those of the PCL-SMPUs with the same $\gamma$ revealed that the heat of fusion is always higher for the PEG-series than those of the PCL-series, implying that the PEG-series may have higher crystallinity than the PCL-series. It is known that the crystallinity of polyols can influence the phase segregation between the soft and the hard segments, and high crystallinity is related to low phase compatibility and poor SME [13].

On the other hand, $\Delta \mathrm{H}_{f}$ increases gradually with IPDI concentration, indicating that the crystallinity of the SMPUs increases, in agreement with our previous work on SMPUs with low molecular weight polyols [20]. It is believed that the reactivity between the polyols and MDI molecules is higher than that between the polyols and IPDI molecules. This results in low crystallinity in the soft segment with a high MDI concentration, and enhances the phase compatibility between the soft and the hard segments, which is better for SME. An increase in IPDI concentration results in a reduction of chemical reactions between MDI and polyol, leading to co-existence of the soft and hard segment macrophases, poor polymerization and poor shape memory effect [20]. Although the transition temperatures are similar to the PCL-SMPUs, the PEG-SMPU samples showed $\Delta H_{f}$ values that were two to three times those of the PCL-SMPUs, indicating that the high molecular weight polyols tend to crystallize more easily than those with low molecular weight. This was further confirmed by the X-ray measurements as shown in Figure 4, where the PEG-SMPUs have higher intensities of the X-ray diffraction peaks than those of the low molecular weight polyol SMPUs.

Figure 4. WAXD curves of the PEG (a) and PCL (b) SMPUs.
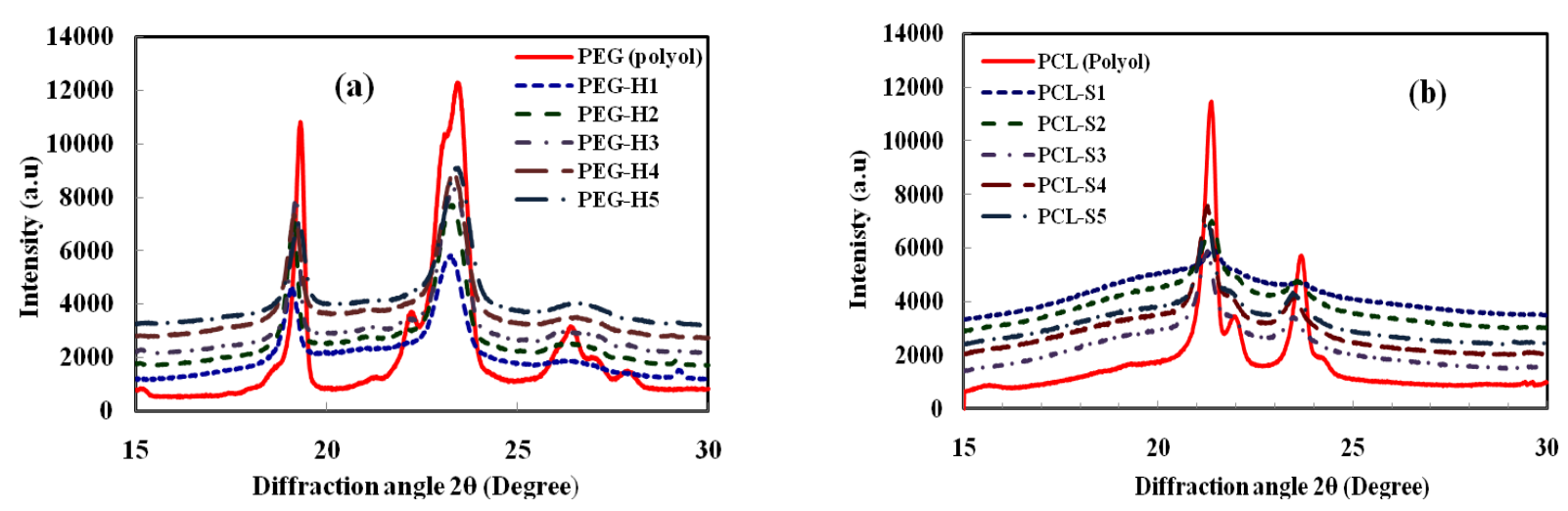

The major peaks in the WAXD curves of both the sample series remained unchanged compared to those of pure polyols. The degree of the crystallinity decreased with the increase in $\gamma$ value, which is in agreement with the conclusion that the polyols determine the crystalline structure of SMPUs, whilst introduction of hard segments only changes the crystallinity of the copolymers, as found in our previous work [20], which affects the phase compatibility.

In brief, the PEG-SMPUs have higher crystallinity and lower cross-link density at soft segment sites than those of the PCL-SMPUs. The results indicate that the PEG-SMPUs may be stiffer with lower elongation at break and possibly poorer shape memory properties. 


\subsection{Thermal Mechanical Properties}

Similar to the PCL-SMPUs, the maximum elongation at break (or the maximum strain) for the PEG-SMPUs varies significantly with $\gamma$ as shown in Figure 5(a,b). The PCL-SMPUs show high maximum strains at break; it is about $930 \%$ for PCL-S1 with $100 \%$ MDI, gradually decreasing to about $192 \%$ as $\gamma$ decreases from $\gamma=1$ to 0.325 . Compared with those of the PCL-SMPUs, the maximum strains of the PEG-SMPUs are less than half of those of the PCL samples with the same $\gamma$. It is clear that the PCL-2000 polyol produces more flexible and elastic SMPUs than the PEG-6000 polyol does. These results are consistent with the observations from both the DSC and WAXD measurements, i.e., the PEG-SMPUs have high crystallinity and are stiff, and their stiffness increases with the IPDI concentration. Inter-molecular interaction at the soft segment sites is reduced as the molecular weight increases, and this may also be responsible for the early break of the PEG-SMPUs [27,28]. Furthermore, PEG-6000 has $\mathrm{N}_{\mathrm{OH}} \sim 18$, much smaller than that of PCL-2000 $\left(\mathrm{N}_{\mathrm{OH}} \sim 55\right)$, providing much less cross-link sites for reaction with the hard segments. Consequently, the PEG-SMPUs have much fewer cross-links, weaker mechanical strength and lower elongation at break. It is also clear from the results shown in Figure 6(a) that a higher molar ratio of MDI to IPDI is better for synthesizing both the sample series with larger maximum strains, consistent with our previous work [20].

Figure 5. Stress vs. strain for PEG (a) and PCL-SMPUs (b) series at ambient temperature.
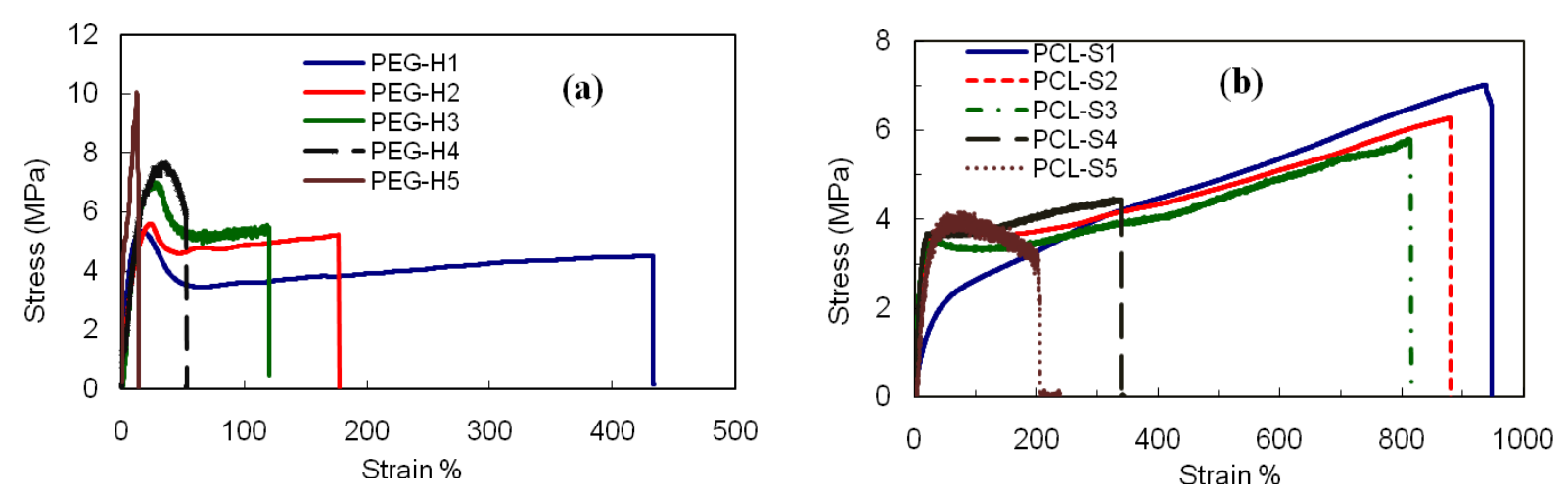

Figure 6. Summary of elongation at break (a) and maximum stress (b) for PEG and PCL SMPUs, respectively, at ambient temperature.
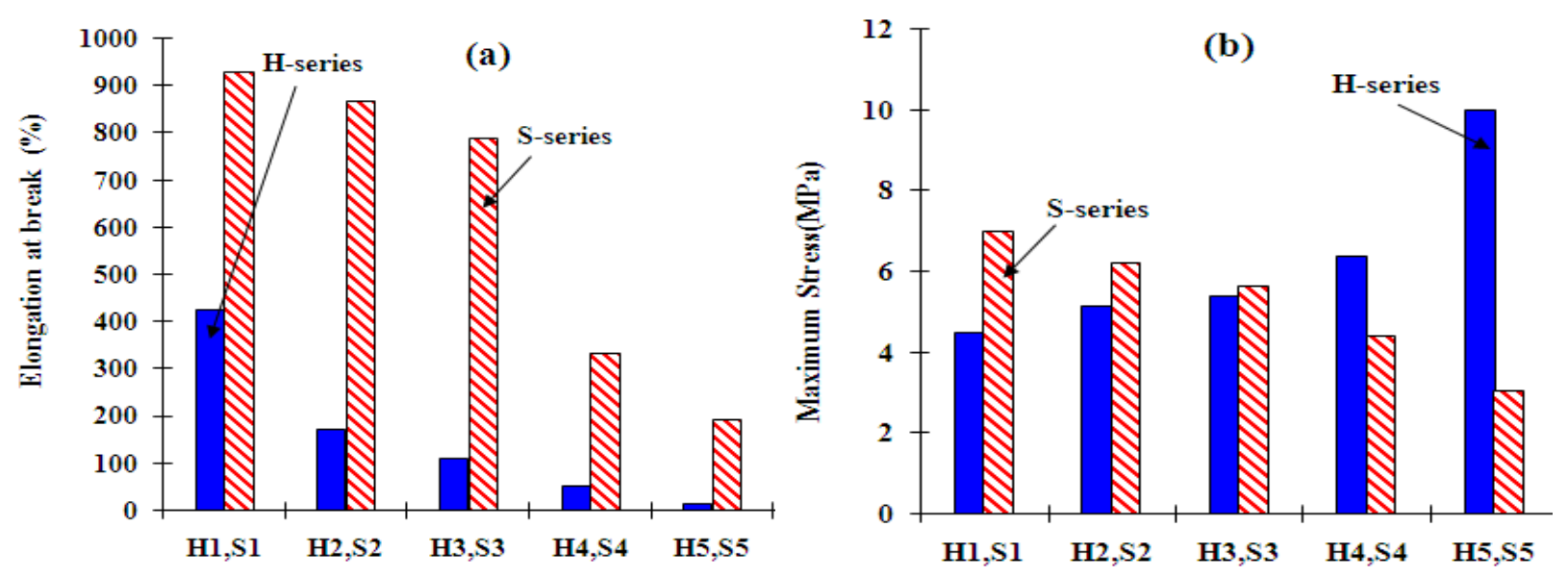
The maximum tensile strength, defined as the stress at which the sample breaks, for both PEG and PCL based SMPUs are summarized in Figure 6(b). The maximum strength of the PEG-SMPUs show a gradual increase with the IPDI content, whereas those of the PCL-SMPUs shows a decrease of the maximum strength with the IPDI content, in agreement with our previous work [20]. This remarkable difference clearly indicates that the chemistry of the polyols plays a vital role in the thermal mechanical behaviour of the SMPUs. This may be due to (a) the resistance of the IPDI to the movement of the soft segment chains, which will enhance the stiffness of the SMPU with a high IPDI concentration [29]; and (b) the high crystallinity of the high molecular weight polyols as discussed above. Stress vs. strain behavior for the PCL-SMPUs shows more elastic behavior, i.e., the stress rapidly increases with strain initially, and then is followed by a linear increase. Whereas, that of the PEG-series shows a clear plastic behavior, i.e., the stress increases with strain initially and then drastically decreases; a typical plastic behavior.

\subsection{Shape Memory Properties}

\subsubsection{Recovery Stress}

Figure $7(\mathrm{a}, \mathrm{b})$ shows the stress and strain relationship of PEG-H1 up to $50 \%$ strain at different thermal cycles, and the corresponding recovery stresses measured from the deformed strips. As expected, the stress increases with strain, and the maximum stress at 50\% strain decreases with thermal cycles. The residual strain increases with thermal cycles is mostly caused by internal molecular rearrangement and chain disentanglements, especially in the first round of extension, and is a typical characteristic of polymers. The recovery stress starts to build up at about $35^{\circ} \mathrm{C}$ (Figure $7(\mathrm{~b})$ ), which is about $10{ }^{\circ} \mathrm{C}$ lower than the transition temperature of the polymer. Above $35{ }^{\circ} \mathrm{C}$, the recovery stress rises rapidly with the temperature. The full recovery stress is developed at a temperature where the sample was pre-deformed, but is much smaller than the maximum stress developed during deformation as shown in Figure 7(a), mostly due to the relaxation of the stress. The recovery stress generated in the 1st cycle test is $0.038 \mathrm{MPa}$, and becomes 0.025 and $0.020 \mathrm{MPa}$ respectively for the $2 \mathrm{nd}$ and the 3rd cycle tests, showing a rapid deterioration of the recovery strength on cyclic test, a typical characteristic for SMPUs due to the accumulation of the broken chains, molecules or linking points and stress relaxation [30].

Figure 7. (a) Stress vs. strain up to an extension of $50 \%$ at $60{ }^{\circ} \mathrm{C}$ and (b) corresponding recovery stress for PEG-H1 sample.
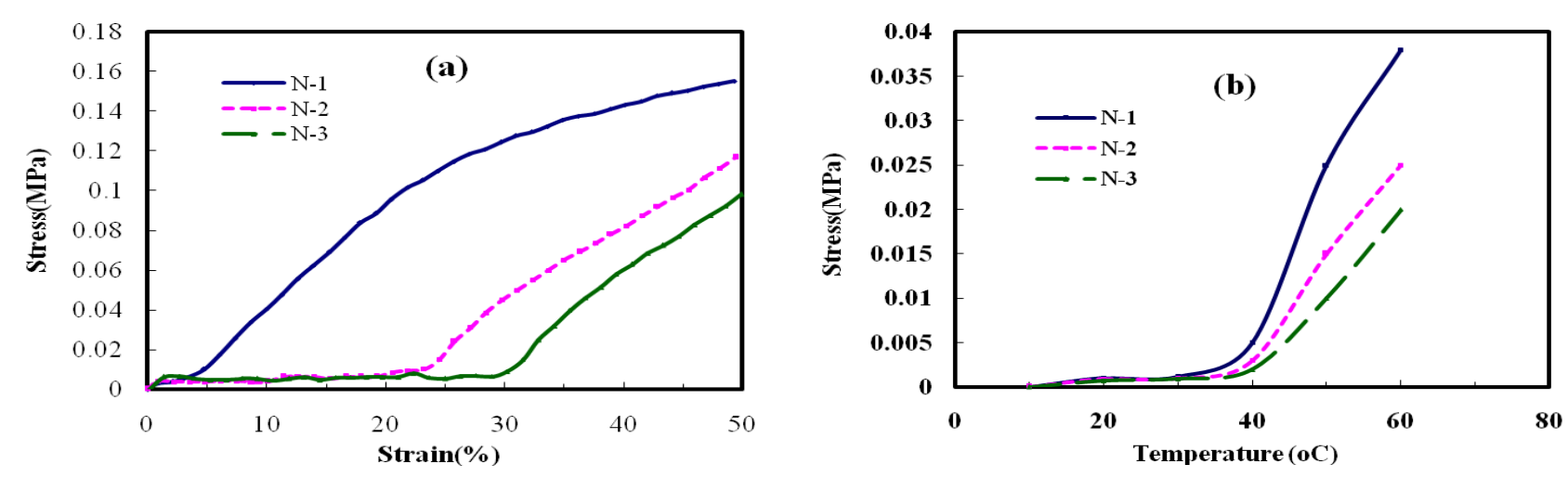
The same cyclic test at an extension of 50\% was applied to the PCL-SMPUs, and the recovery stress for both sample series are summarized in Figure 8 (results from H5 and S5 samples were not shown as H5 could not be extended up to $50 \%$ as shown in Figure 5(a)). It is clear that the PEG-SMPUs produce lower recovery stresses which are attributed to lower cross-link density, longer soft segment chains (higher molecular weight) and weaker intermolecular interactions. The value of the recovery stress for each sample series increases as $\gamma$ decreases, consistent with the results of the SMPUs with low molecular weight polyols [20]. This further indicates that increase of the IPDI content enhances the stiffness of the SMPUs, in agreement with the DSC and WAXD test results where the heat of fusion and crystallinity also increase with the IPDI concentration.

Figure 8. Summary of recovery stress of different SMPUs at $60{ }^{\circ} \mathrm{C}$ and $50 \%$ strain.

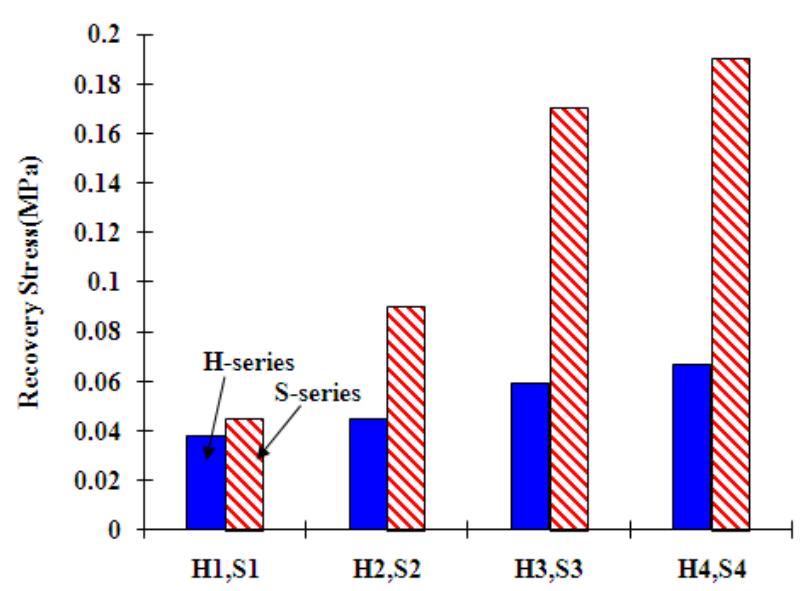

\subsubsection{Shape Recovery Ratio and Shape Fixity}

The shape recovery ratio of the PEG-SMPUs, shown in Figure 9(a), varies from 98 to 82\%, depending on the molar ratio $\gamma$. PEG-H1 has the highest shape recovery ratio of $98 \%$, and that of PEG-H4 is only $82 \%$. The PEG-SMPUs show a better recovery ratio compared to those of the PCL-SMPUs, indicating that the high $\mathrm{M}_{\mathrm{W}}$ polyol SMPUs are more flexible at elevated temperature, in contrast to the room temperature thermal mechanical properties shown above. This may be attributed to the longer molecular chains of the high $\mathrm{M}_{\mathrm{W}}$ polyol soft segment, easy deformation of the SMPUs at above $\mathrm{T}_{\mathrm{g}}$ as compared to low molecular weight polyols.

Figure 9. Shape recovery and shape fixity of the PEG (a) and PCL SMPUs (b) at $60{ }^{\circ} \mathrm{C}$ and 50\% strain.
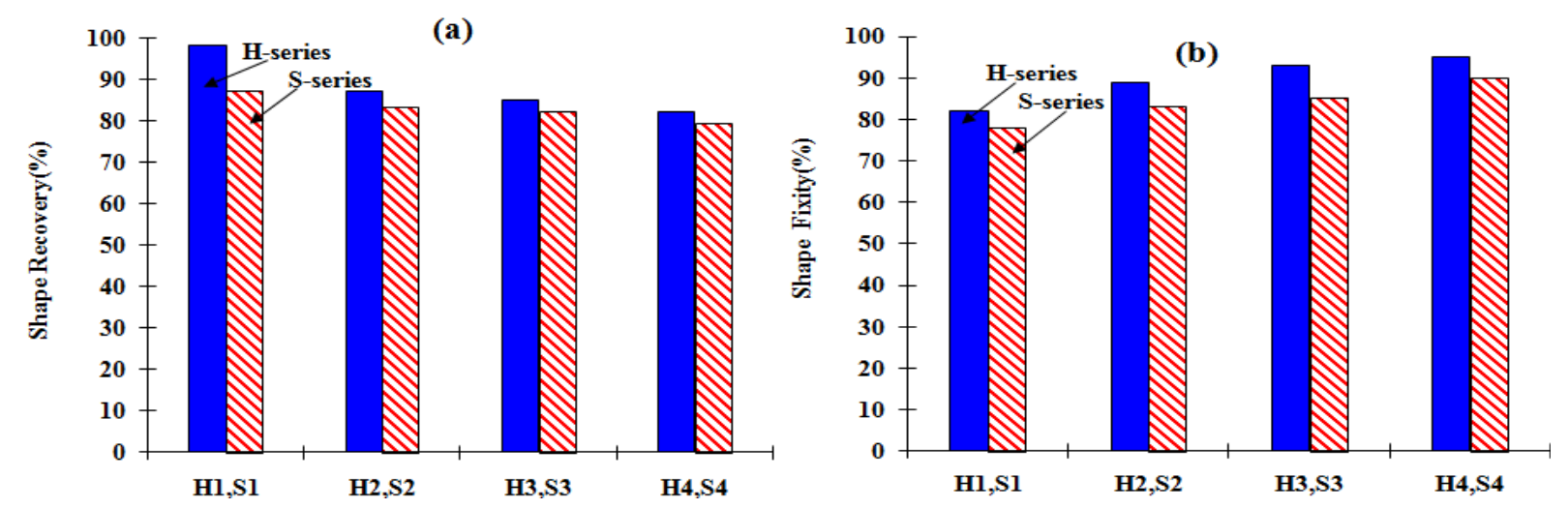
Figure 9(b) summarizes the shape fixity for both series of the samples. The shape fixity is in the range of $82 \sim 95 \%$ for the PEG-SMPUs. It increases with the decrease of $\gamma$. PEG-H1 with the highest $\gamma$ shows the lowest shape fixity of about $82 \%$, while that of PEG-H4 with $\gamma=0.5$ is $\sim 95 \%$. The shape fixity of the PEG-SMPUs is higher than PCL-SMPUs due to the high stiffness and high crystallinity as discussed above. Similarly, introduction of the IPDI restricts the segmental and short-range conformational movements of the soft segments, which leads to a quick fixing of the soft segment chains during cooling below the transition temperature. This trend is in agreement with the observation made for the PCL-SMPUs, which have a slightly lower fixity than the PEG-SMPUs with the same MDI to IPDI molar ratios. Generally, the high recovery ratio is always accompanied with poor fixity for shape memory polymers, and high recovery ratio and high fixity are the most desirable properties for SMPs. The PEG-SMPUs have a high recovery ratio and high fixity as well as high maximum stress, demonstrating their excellent shape memory effect as compared to those of the PCL-SMPUs, if the maximum strain of $\sim 400 \%$ is allowed which is the normal case for typical applications.

\subsection{Physical Shape Recovery Test}

Shape recovery speed is another key property of shape memory polymers, which refers to the time for a deformed SMP sample to recover their original shape. The recovery or actuation times for SMPs are in the range of about one minute to over one hundred minutes [27], much longer than those of shape memory alloys. For most applications of SMPs, shape recovery should be as quick as possible, so that the SMPs can be used to develop microactuators and devices which can be activated easily by external heat or light in the shortest time. For example, SMPs with high speed and low $T_{\mathrm{g}}$ have good potentials for medical applications such as drug delivery systems, polymer-based stents and micro-surgery tools or sutures. In this study, the physical shape recovery tests were performed to study the recovery behavior and recovery speed of the SMPUs. The samples with a thickness of $0.5 \mathrm{~mm}$, a width of $3 \mathrm{~mm}$ and a length of $\sim 70 \mathrm{~mm}$, were used for the experiments with one example shown in Figure 10. The SMPU sample was initially stretched to $100 \%$ at $60{ }^{\circ} \mathrm{C}\left(>T_{\mathrm{g}}\right)$, and the deformed shape was fixed by cooling it to room temperature $\left(\sim 20^{\circ} \mathrm{C}\right)$. The sample was then put on a hot plate set at $60{ }^{\circ} \mathrm{C}$ and its subsequent shape changes over time were recorded.

Figure 10. Shape recovery of the $100 \%$ extended PEG-H3 at $60{ }^{\circ} \mathrm{C}$. The shape recovery takes place immediately when heated, and completes the process within $20 \mathrm{~s}$.

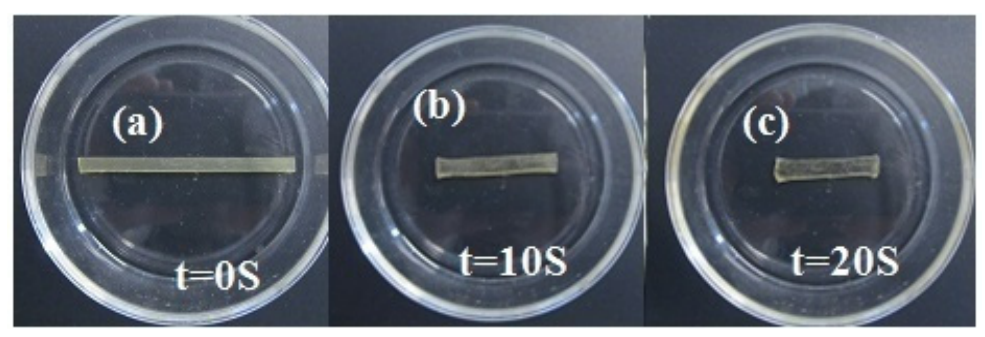

It was found that the PEG-SMPU samples start to shrink (recovering the original state) instantly once they are put on the hotplate. The PEG-H3 samples show about $90 \%$ recovery in less than $10 \mathrm{~s}$, and a full recovery process is completed within $\sim 20 \mathrm{~s}$, demonstrating an excellent actuation capability. This is significantly different from those of the PCL-SMPUs, all of which show an actuation 
time $>50 \mathrm{~s}$ [20], though they were in a roll form which may have a non-uniform temperature distribution. An example of the PCL-S3 sample (100\% extension) is shown in Figure 11. It shows that the sample takes a long time to recover, with $<80 \%$ recovery after $50 \mathrm{~s}$. The results clearly demonstrate that the high molecular weight PEG-SMPUs have a much higher actuation speed as compared to those of the PCL-SMPUs, and may be a good choice for polymer-based microactuator applications [31]. Further work is needed to investigate SMPUs with other high molecular weight polyols.

Figure 11. Shape recovery of the extended PCL-S3 sample at $60{ }^{\circ} \mathrm{C}$. The shape recovery takes place slowly and is completed up to $\sim 80 \%$ after $50 \mathrm{~s}$; a much slower rate than that recorded for PEG-SMPUs.

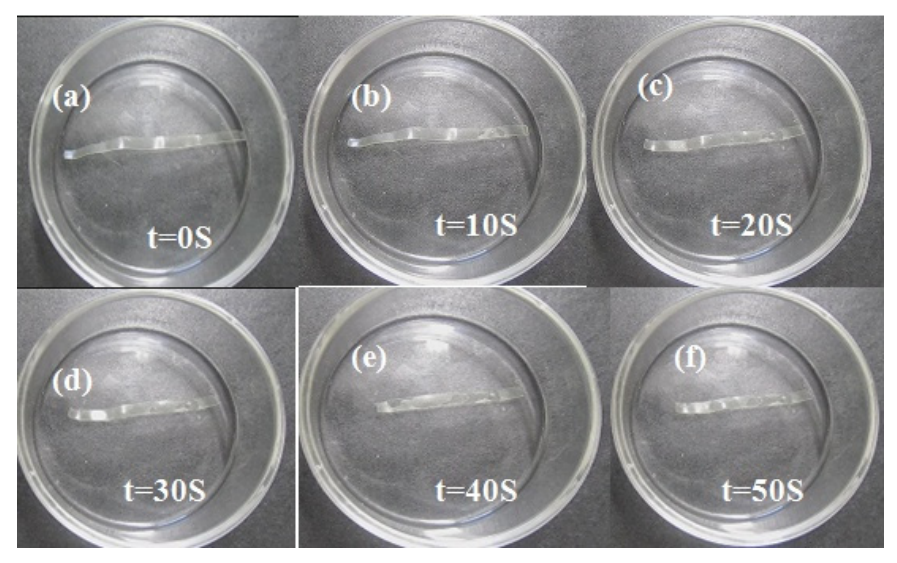

\section{Conclusions}

SMPUs with high molecular weight polyol PEG-6000 were successfully synthesized with various combinations of diisocyanates as a hard segment, and the results were compared with SMPUs with low molecular weight PCL-2000 polyol. The thermal stability, thermal mechanical properties, crystallinity and shape memory effects of the SMPUs were investigated. The conclusions can be summarized as follows;

- The PEG-SMPUs show a slightly better thermal stability than PCL-SMPUs. Both sample series have transition temperatures between $40 \sim 50{ }^{\circ} \mathrm{C}$, suitable for medical device applications.

- The PEG-SMPUs have a higher crystallinity and lower phase compatibility than those of PCL-SMPU samples. With the increase of IPDI concentration, the crystallinity increases, leading to deteriorated phase compatibility and shape memory effect for both the sample series.

- The PCL-SMPUs have larger elongations at break and recovery stresses than those of the PEG-SMPUs, i.e., high mechanical strength at room temperature. Longer molecular chains, lower cross-link density and higher crystallinity are believed to be responsible for the weaker thermal mechanical properties of the PEG-SMPUs.

- The PEG-SMPUs have a higher recovery ratio, a higher fixity and a much shorter shape recovery time, and demonstrate superior SME effect as compared to the PCL-SMPUs. SMPUs with high molecular weight polyol can be excellent candidates for applications in engineering and as microactuators, in particular when fast actuation is required. 


\section{Acknowledgement}

The authors would like to acknowledge partial financial support from the Engineering and Physical Sciences Research Council under grant No. of EP/F06294X/1 and The leverhulme Trust under grant No. of F/01431, The Knowledge Centre for Materials Chemistry under grant No. of X00680PR, Royal Academy of Engineering-Research Exchanges with China and India Awards, Royal Society-Research Grant (RG090609) as well as Carnegie Trust Funding.

\section{References}

1. Tobushi, H.; Hashimoto, T.; Ito, N.; Hayashi, S.; Yamada, E. Shape fixity and shape recovery in a film of shape memory polymer of polyurethane series. J. Intell. Mater. Syst. Struct. 1998, 9, 127-136.

2. Lendlein, A.; Langer, R. Biodegradable, Elastic shape-memory polymers for potential biomedical applications. Science 2002, 296, 1673-1677.

3. Tobushi, H.; Haray, H.; Yamaday, E.; Hayashiz, S. Thermomechanical properties in a thin film of shape memory polymer of polyurethane series. Smart Mater. Str. 1996, 5, 483-491.

4. Liang, C.; Rogers, C.A.; Malafeew, E. Preliminary investigation of shape memory polymers and their hybrid composites. Smart Struct. Mater. 1991, 24, 97-103.

5. Hayashi, S.; Ishikawa, N.; Jiordano, C. High moisture per-meability polyurethane for textile application. J. Coated Fabrics 1993, 23, 74-83.

6. Huang, W.M.; Yang, B.; Zhao, Y.; Ding, Z. Thermo-moisture responsive polyurethane shape-memory polymer and composites: A review. J. Mater. Chem. 2010, 20, 3367-3381.

7. Wei, Z.G.; Sandstrom, R.; Miyazaki, S. Review shape-memory materials and hybrid composites for smart systems. J. Mater. Sci. 1998, 33, 3745-3762.

8. Chun, B.C.; Cha, S.H.; Chung, Y.C.; Cho, J.W. Enhanced dynamic mechanical and shape-memory properties of a poly(ethylene terephthalate)poly(ethylene glycol) copolymer crosslinked by maleic anhydride. J. Appl. Polym. Sci. 2002, 83, 27-37.

9. Lee, H.S.; Wang, Y.K.; Macknight, W.J.; Hsu, S.L. Spectroscopic analysis of phase-separation kinetics in model polyurethanes. Macromolecules 1988, 21, 270-273.

10. Xia, H.S.; Song, M.; Zhang, Z.Y.; Richardson, M. Microphase separation, stress relaxation, and creep behavior of polyurethane nanocomposites. J. Appl. Polym. Sci. 2007, 103, 2992-3002.

11. Coleman, M.M.; Lee, K.H.; Skrovanek, D.J.; Painter, P.C. Hydrogen-bonding in polymers. 4. Infrared temperature studies of a simple polyurethane. Macromolecules 1986, 19, 2149-2157.

12. Seymour, R.W.; Allegrezza, A.E.; Cooper, S.L. Segmental orientation studies of block polymers. i. hydrogen-bonded polyurethanes. Macromolecules 1973, 6, 896-908.

13. Lee, B.S.; Chun, B.C.; Chung, Y.C.; Sul, K.I.; Cho, J.W. Structure and thermomechanical properties of polyurethane block copolymers with shape memory effect. Macromolecules 2001, 34, 6431-6437.

14. Lee, H.S.; Kim, J.W.; Kim, K.B. Shape memory polyurethanes having crosslinks in soft and hard segments. Smart. Mater. Struct. 2004, 13, 1345-1350.

15. Kim, B.K.; Lee, S.Y. Polyurethanes having shape memory effects. Polymer 1996, 37, 5781-5793. 
16. Li, F.; Zhang, X.; Hou, J.; Xu, M.; Luo, X.; Ma, D.; Kim, B.K. Studies on thermally stimulated shape memory effect of segmented polyurethanes. J. Appl. Polym. Sci.1997, 64, 1511-1516.

17. Mondal, S.; Hu, J.L. Studies of shape memory property on thermoplastic segmented polyurethanes: Influence of PEG 3400. J. Elastomer \& Plastic. 2007, 39, 81-91.

18. Chen, S.; Hu, J.; Liu, Y.; Liem, H.; Zhu, Y.; Meng, Q. Effect of molecular weight on shape memory behavior in polyurethane films. Polym. Int. 2007, 56, 1128-1134.

19. Hu, J.; Yang, Z.; Yeung, L.; Ji, F.; Liu, Y. Crosslinked polyurethanes with shape memory properties. Polym. Int. 2005, 54, 854-859.

20. Ahmad, M.; Luo, J.K.; Xu, B.; Purnawali, H.; King, P.J.; Chalker, P.; Fu, Y.Q.; Huang, W.M.; Miraftab, M. Synthesis and characterization of polyurethane based shape memory polymers. Macromol. Chem. Phys. 2011, 202, 592-602.

21. Lendlein, A.; Kelch, S. Shape memory polymers. Angew Chem. Int. Edit. 2002, 41, 2034-2057.

22. Tobushi, H.; Hara, H.; Yamada, E.; Hayashi, S. Thermomechanical properties in a thin film of shape memory polymer of polyurethane series. Smart Mater. \& Struct. 1996, 5, 483-496.

23. Tien, Y.I.; Wei, K.H. The effect of nano-sized silicate layers from montmorillonite on glass transition, dynamic mechanical, and thermal degradation properties of segmented polyurethane. Appl. Polym. Sci. 2002. 86, 1741-1748.

24. Hayashi, S.; Kondo, S.; Kapadia, P.; Ushioda, E. Room-temperature-functional shape-memory polymers. Plast. Eng. 1995, 51, 29-31.

25. Huang, W.M. Thermo-moisture responsive polyurethane shape memory polymer for biomedical devices. J. Open Med. Device 2010, 2, 11-19.

26. Ahmad, M.; Luo, J.K.; Miraftab, M. Feasibility study of polyurethane shape-memory polymer actuators for pressure bandage application. Sci. Technol. Adv. Mater. 2012, 13, 015006-015013.

27. Cho, J.W.; Jung, Y.C.; Chung, Y.C.; Chun, B.C. Improved mechanical properties of shape-memory polyurethane block copolymers through the control of the soft-segment arrangement. J. Appl. Polym. Sci. 2004, 93, 2410-2015.

28. Wilkes, G.L.; Wildnauer, R. Kinetic behavior of the thermal and mechanical properties of segmented urethanes. J. Appl. Phys. 1975, 46, 4148-4152.

29. Bassi, M.; Tonelli, C.; Di, A.M. Glass transition behavior of a microphase segregated polyurethane based on PFPE and IPDI: A calorimetric study. Macromolecules 2003, 36, 8015-8023.

30. Lendlein, A.; Jiang, H.; Junger, O.; Langer, R. Light-induced shape memory polymer. Nature 2005, 434, 879 .

31. Small, W.; Wilson, T.S.; Benett, W.J.; Loge, J.M.; Maitland, D.J. Laser-activated shape memory polymer intravascular thrombectomy device. Opt. Express 2005, 13, 8204-8213.

(C) 2012 by the authors; licensee MDPI, Basel, Switzerland. This article is an open access article distributed under the terms and conditions of the Creative Commons Attribution license (http://creativecommons.org/licenses/by/3.0/). 